\title{
Policy \\ ATA Practice Guidelines for Live, On-Demand Primary and Urgent Care
}

Frances Gough, MD, ${ }^{1}$ Sunil Budhrani, $M D, M P H, M B A^{2}$

Ellen Cohn, PhD, ${ }^{3}$ Alan Dappen, $M D_{1}^{4}$

Cindy Leenknecht, MS, ACNS-BC, ${ }^{5}$ Bill Lewis, $M D_{1}^{6}$

Deborah Ann Mulligan, MD, Deborah Randall, $J D_{1}^{8}$

Karen Rheuban, MD, ${ }^{9}$ Lisa Roberts, PhD, ${ }^{10}$ Terrance J. Shanahan, JD, ${ }^{11}$

Kathy Webster, $M D_{,}{ }^{12}$ Elizabeth A. Krupinski, $P h D,{ }^{13}$

Rashid Bashshur, PhD, ${ }^{14}$ and Jordana Bernard, MBA ${ }^{15}$

${ }^{7}$ Molina Healthcare of Washington, Seattle, Washington.

${ }^{2}$ CareClix, Tysons Corner, Virginia.

${ }^{3}$ School of Health and Rehabilitation Sciences, University

of Pittsburgh, Pittsburgh, Pennsylvania.

${ }^{4}$ DocTalker Family Medicine, Vienna, Virginia.

${ }^{5} \mathrm{SCL}$ Health System, Denver, Colorado.

${ }^{6}$ Concentra, Phoenix, Arizona.

${ }^{7}$ MDLIVE, Institute for Child Health Policy, Nova Southeastern

University, Ft. Lauderdale, Florida.

${ }^{8}$ Deborah Randall Consulting, Washington, D.C.

${ }^{9}$ University of Virginia Center for Telehealth, University of

Virginia Health System, Charlottesville, Virginia.

${ }^{10}$ AMC Health, New York, New York.

"The O'Neil Group, LLC, Washington, D.C.

${ }^{12}$ Loyola University Medical Center, Maywood, Illinois.

흘 ${ }^{13}$ Department of Medical Imaging, University of Arizona, Tucson,

A Arizona.

骂 ${ }^{14}$ University of Michigan Health System, Ann Arbor, Michigan.

${ }^{15}$ American Telemedicine Association, Washington, D.C.

\section{Preamble}

he American Telemedicine Association (ATA) is a memas well as the public and private sectors in promoting the safe and effective use of telemedicine to promote the health and wellbeing of people.

ATA has embarked on a mission to establish practice guidelines in many areas of telemedicine practice to promote patient safety and uniformity and quality of services provided via telemedicine. The guidelines were developed by panels of experts in this field and are designed to assist providers of care in adhering to ethical standards and sound business practices. They are intended to serve as guides for patients and their caregivers in assuring their rights and protecting their health.

The development of these guidelines entailed a rigorous process of peer review and analysis to ensure their appropriateness, relevancy, consistency, and comprehensiveness. They were enacted after full review and approval by the Board of Directors. In view of changing circumstances and developments, these guidelines are reviewed periodically and updated as indicated.

Compliance with these guidelines alone will not guarantee accurate diagnoses or successful outcomes. The practitioner should rely on his or her best professional experience and expertise when faced with unexpected circumstances or new developments in technology. When this occurs, the practitioner is strongly advised to document his or her rationale in the patient record.

The framers of these guidelines do not purport to establish legal standards for telemedicine services but focus on the quality, safety, and effectiveness of telemedicine encounters.

\section{Scope}

These guidelines cover the provision of patient-initiated primary and urgent care services by licensed healthcare providers using real-time, interactive technologies, including mobile devices. Healthcare providers include individual practitioners, group and specialty practices, hospitals and healthcare systems, triage or call centers, and other licensed providers of telemedicine services. They do not address communications between healthcare professionals and patients via short message service, e-mail, social network sites, or online "coaching," or the use of telemedicine for primary care when facilitated by a provider connecting to another provider.

The guidelines address three aspects of service delivery: clinical, technical, and administrative. Under each set, the guidelines are classified into three levels of adherence: "shall" indicates required action whenever feasible and/or practical; "shall not" indicates a proscription or action that is strongly advised against; and "should" indicates a recommended action without excluding others. "May" indicates pertinent actions that may be considered to optimize the telemedicine encounter. These indications are found in bold throughout the document.

ATA urges health professionals using telemedicine in their practices to familiarize themselves with these guidelines and with other position statements or standards from their professional organizations and societies and incorporate them into telemedicine practice. They pertain to the United States when both patient and provider are within the United States. Others may use them at their discretion.

\section{Introduction}

Telemedicine enables providers to extend their reach and to improve their efficiency and effectiveness while still maintaining highquality care and attention to patient safety. These guidelines pertain to telemedicine in primary and urgent care encounters initiated by patients. As with the practice of medicine in general, users must 


\section{GOUGH ET AL.}

adhere to strict ethical and professional standards to assure quality of care and patient safety. ATA has developed a series of guidelines including Core Standards for Telemedicine Operations. ${ }^{1}$ This guideline focuses on the use of telemedicine services to connect providers and patients in the delivery of primary and urgent care.

The development of these guidelines followed a rigorous process of evidence review and expert assessment of more than 600 studies regarding the practice of telemedicine in primary and urgent care. Several studies within and outside the United States have demonstrated the safety, efficacy, and quality of telemedicine in primary and urgent medical care for uncomplicated conditions. ${ }^{2-6}$ These studies report outcomes often on par with in-person encounters, high patient satisfaction, and reduced costs of care, depending on the attributes of the intervention and the metrics studied..$^{3-5,7-23}$

Practitioners must be mindful of all relevant statutes and regulations when choosing to provide care to patients via telemedicine, and in particular when considering prescribing medications.

\section{Telemedicine in the Practice of Primary and Urgent Care}

For the purposes of this document, primary and urgent care is defined as the delivery of basic nonspecialty care outside a hospital emergency department when a patient is deemed in need of immediate medical attention. This type of care is typically unscheduled and episodic and is not always provided by the patient's regular primary care provider. Primary care typically implies a longer-term relationship between patient and provider, wherein the provider is usually familiar with the patient's health history.

Both acute and chronic conditions may present with symptoms that range from mild to severe. Examples of acute medical conditions that may be managed effectively by video-based telemedicine and, as appropriate, other interactive technologies supported by peripheral devices and ancillary tests necessary to establish a diagnosis include uncomplicated cases of allergy/asthma, chronic bronchitis, conjunctivitis, genitourinary conditions, low back pain, otitis media, rashes, and upper respiratory tract infections. ${ }^{3,5,6,9,10,24}$ Chronic medical conditions addressed by telemedicine within primary care practices may include mental illness and behavioral health, chronic obstructive pulmonary disease, congestive heart failure, diabetes, and hypertension. ${ }^{8,11,12,25-31}$ The virtual medium is also an appropriate tool for consultations regarding prevention and wellness services such as immunizations, smoking cessation, diet, and physical activity.

Prescribing is generally accepted (given local and federal regulations) within the context of real-time videoconferencing sessions when information can be provided that approximates the in-person exam. Prescribing is also generally accepted based on telephone-only consultations with a patient where there is a prior relationship by the provider or with providers offering weekend coverage with access to the patient's medical record. However, for prescribing based on a telephone session with a patient with no prior relationship and no medical record, there are differences of opinion and few independent, validated studies. One recent study, conducted in a health system outside of the United States (in Denmark), concluded that prescriptions made during telephone consultations conducted under that system were feasible and that practitioners who had access to the patient's medical record were conservative in the encounters, being less likely to offer prescriptions when cases are severe or involve children, ${ }^{32}$ with no differences in prescription rates between contacts due to new episodes and exacerbation of chronic disease. Antibiotics, analgesics, and medications for the respiratory system are the most commonly prescribed drugs. ${ }^{32}$ Other published data, however, have demonstrated higher rates of prescribing for patients with complaints of urinary tract symptoms evaluated and treated via non-videobased visits without laboratory testing. ${ }^{30}$ For isolated patients with no other means to access a provider, an audio-based consult may be the only alternative. The prudent practitioner should be aware of and follow all relevant statutes and regulations regarding the modality of technology used when choosing to provide care to patients via telemedicine, and in particular when considering prescribing medications.

Despite some overlap between acute and chronic diseases, there are several common attributes of primary/urgent care in traditional, in-person practice and video-based telemedicine, including timely service, a trust relationship, and opportunity for follow-up. These are identified in the primary care medical home model. ${ }^{27,33,34}$ The Agency for Healthcare Research and Quality defines the medical home not simply as a location but as an organization that delivers the core functions of primary healthcare. ${ }^{35}$

\section{Practice Guidelines}

Many conditions lend themselves to a virtual visit as defined in this document. Currently there is a growing body of evidence regarding the effectiveness of video- and audio-based interventions for a variety of acute and chronic conditions seen in primary care, such as diabetes, asthma, heart failure, and hypertension. 5,6,8-12,25-31,34,36-38 Typically these include conditions for which there is a reasonable level of certainty in establishing a diagnosis and generating a treatment plan, especially when visual information coupled with access to a medical record with diagnostic studies and imaging is available., ${ }^{5,19,30}$

In general, conditions that are not suitable for telemedicine are those for which an in-person visit is required to evaluate the patient because of the severity of presenting symptoms, the necessity of haptic information, the need for protocol-driven procedures, or the need for aggressive interventions. Other circumstances that are not suitable for telemedicine include some patients with cognitive disorders, intoxication, language barriers, emergency situations that warrant escalation to an emergency room visit or 911, or when patients do not have the requisite technology to complete a virtual visit.

These practice guidelines identify primary and urgent care services that current information indicates can be provided safely and effectively using telemedicine. They are not intended to substitute for the independent medical judgment, training, and skill of the practitioner. Therefore, providers shall exercise their professional judgment when deciding whether or not to use telemedicine, taking into account the patient's condition, mitigating circumstances, available 


\section{ATA PRACTICE GUIDELINES FOR LIVE, ON-DEMAND CARE}

resources, and their own comfort level and expertise in using telemedicine. Providers shall be aware of all relevant state and federal regulations related to the use of telemedicine to include those that govern prescribing as it relates to the establishment of a doctorpatient relationship. In addition, practitioners shall be aware of relevant practice guidelines developed by the specialty societies as they relate to both in-person and telehealth practice.

\section{PRELIMINARY CONSIDERATIONS}

A. Regulatory and licensure requirements. Providers shall follow $\therefore$ federal, state, and local regulatory and licensure requirements related to their scope of practice and shall abide by state board and specialty training requirements. Providers shall ensure that the patient is physically located in a jurisdiction in which the provider is duly

licensed and credentialed. Providers shall practice within the scope of their licensure and shall observe all applicable state and federal legal $\stackrel{\sim}{\infty}$ and regulatory requirements.

B. Informing and educating the patient. Prior to the initiation of a telemedicine encounter, the provider or designee shall inform and . educate the patient (either in writing or verbally) about the nature of telemedicine service compared with in-person care. This shall include discussion of the nature of a telemedicine encounter, timing of service, record keeping, scheduling, privacy and security, potential I. risks, mandatory reporting, the credentials of the distant site provider, and billing arrangements. The information shall be provided in simple language that can be easily understood by the patient. This is particularly important when discussing technical issues like encryption or the potential for technical failure.

More specifically, this information shall include the limits to confidentiality in electronic communication, an explicit emergency plan (particularly for patients in settings without access to clinical staff), a process by which patient information will be documented and stored, the potential for technical failure, procedures for coordination of care with other professionals, a protocol 응 for contact between visits, prescribing policies including local $\underset{\nabla}{*}$ and federal regulations and limitations, and the conditions under Fhich telemedicine services may be terminated and a referral made o to in-person care.

Finally, the provider or designee should set appropriate expectations regarding the telemedicine encounter, including, for example, prescribing policies, scope of service, communication, and follow-up. To reduce the risk of overprescribing, the provider shall follow evidence-based guidelines and all federal, state, and local regulations.

C. Physical environment. The provider shall determine the minimal acceptable levels of privacy, lack of distraction and background noise, and other environmental conditions that may affect the quality of the encounter, in particular when video-based services are offered. The provider's and the patient's room/environment should ensure privacy to prevent unauthorized access. Seating and lighting should be designed for both comfort and professional interaction. Both provider and patient should be visible and heard. Patients receiving care in non-traditional settings should be informed of the importance of reducing background light from windows or light emanating from behind them. Both provider and patient cameras should be placed on a secure, stable platform to avoid wobbling and shaking during the videoconferencing session. To the extent possible, the patient and provider cameras should be placed at the same elevation as the eyes with the face clearly visible to the other person.

D. Referrals and emergency resources. The provider shall have an emergency or contingency plan that is communicated to the patient in advance of the telemedicine encounter.

The provider should be familiar with, or have access to, available medical resources in proximity to the patient in order to make referrals or request transfers when indicated.

E. Cultural competence. Telemedicine providers and their staff shall deliver services in a culturally competent manner that takes into account the patient's age, disability status, ethnicity, language, gender, gender identity and sexual orientation, geographical location, language, religion, and socioeconomic status.

Provider and patient or patient/representative should be able to converse in a language comfortable and familiar to both parties, allowing the provider to obtain a clear history and the patient/ representative to understand the recommendations provided. If necessary, a translator (or signer for deaf/hearing impaired patients) should be used.

\section{TELEMEDICINE MANAGEMENT OF THE PATIENT}

Telemedicine providers shall determine the appropriateness of telemedicine on a case-by- case basis, whether or not a telemedicine visit is indicated, and what portion of the examination must be performed and documented in conformance with appropriate standards in evaluating the patient. Wherever possible, diagnostic interventions should be supported by high-quality evidence. Where evidence is lacking, providers shall use their professional judgment, experience, and expertise in making such decisions. Conditions for use of telemedicine are likely to change to reflect new evidence from future research and the evolution of the enabling technology.

Telemedicine providers shall be cognizant of establishment of a provider-patient relationship in the context of a telemedicine encounter, whether using synchronous or asynchronous modes of communication/interaction makes a difference, and they shall proceed accordingly with an evidence-based standard of care. If not previously established, a provider-patient relationship may be established where the provider is guiding the process of care. The provider shall use his or her professional judgment and conform to all state and federal regulations in determining whether a providerpatient relationship has been established and whether it is sufficient to proceed with an encounter and make diagnostic and treatment decisions including prescribing. In the event the patient does not have a primary care provider, the provider should recommend options to assure continuity of care for the patient. Provider practices 


\section{GOUGH ET AL.}

should establish standard operating procedures and workflows for telemedicine visits consistent with prevailing norms.

Telemedicine management of the patient may involve establishment of a diagnosis and treatment plan, or it may result in a referral to a medical facility for further evaluation and/or treatment.

Clinical protocols should be developed for live, on demand services. Such protocols are beyond the scope of these guidelines, and practitioners are advised to review specialty society guidelines and the evidence published in the peer-reviewed literature. However, protocols should include the following components:

- Named condition and corresponding ICD code

- Scope of condition amenable to treatment by telemedicine based on medical evidence or, at a minimum, precedent for successful management based on peer-reviewed guidelines or expert opinion

- The mode of intervention required to diagnose and treat the condition (i.e., under what circumstance and regulatory framework is telephonic care adequate, is videoconferencing required, are peripheral devices needed or other diagnostic tests, or is an in-person visit needed)

- Documentation required to appropriately assess the patient's condition, including history and any video-based examination including required components needed to visualize, demonstrate, or test

- Parameters under which the condition can be treated

- Parameters under which the condition may not be treated and require referral to alternate modes of management

- Parameters under which prescribing can and cannot be done.

A. Patient evaluation. Patient examination should be commensurate with the level of assessment required to manage a patient, taking into consideration the technical quality and extent of information that may be elicited remotely. This evaluation should be supported by clinical history, access to the patient's medical record where possible, diagnostic data (e.g., obtained via self-report or access to store-and forward databases) and laboratory test results, and peripheral devices for patient physical examination when appropriate.

Audio-based evaluation may be used for consultation, if and only if the evaluation, diagnosis, and treatment of conditions can be made reliably on the basis of complete medical history, with a full understanding of presenting symptoms reported by the patient or caregiver, and be consistent with established clinical protocols, state and federal laws, and regulations related to audio-based evaluations, in particular when such evaluation results in prescribing.

The telemedicine provider should obtain all the data necessary for a diagnosis and treatment plan. Necessary items include:

- Identifying information

- Source of the history

- Chief complaint(s)

- History of present illness (including location, description, size, quality, severity, duration, timing, and context-modifying factors)
- Associated signs and symptoms

- Past medical history

- Family history

- Personal and social history

- Medication review

- Allergies including medication, nature, and severity of reaction

- Detailed review of symptoms

- Provider-directed patient self-examination to include the use of peripheral devices as appropriate.

Documentation shall be performed following each patient encounter and shall be maintained in a secure, Health Insurance Portability and Accountability Act (HIPAA)-compliant form and location (e.g., paper/fax, server, cloud).

Following every visit, the provider shall communicate results of the encounter to the patient's primary care provider or other specialty providers using secure methods (e.g., e-mail/fax, secure e-mail, transmission to electronic medical record), as well as to the patient, unless the patient has requested a limitation on such communication. An appropriate disposition shall also be discussed with the patient including any required follow-up and discussion of clinical signs that would signify a significant escalation. Laboratory tests, including diagnostics ordered in the usual course of evaluation, shall be followed up in a timely manner with the patient and any additional providers as needed.

B. Physical examination. The provider shall perform a virtual physical examination as indicated by the patient complaint and medical history and other relevant information reported by the patient, conforming to the standards of medical practice and provided by a credentialed and qualified practitioner. This examination may include a demonstration or an explicit physician-guided self-examination, which, as appropriate, may include peripheral devices. Where additional diagnostic testing is required to confirm the diagnosis, the provider shall recommend to the patient that such testing be performed in accordance with standards of medical care.

\section{QUALITY}

The provider shall employ a coordinated quality improvement program or clinical oversight process.

A. Quality review. Quality review should be conducted on a periodic basis to identify specific risks and qualify failures. It should include assessment of:

- equipment or connectivity failures

- number of attempted and completed visits

- patient and provider satisfaction with the virtual visit

- patient or provider complaints related to the virtual visits (e.g., via surveys)

- measures of clinical quality such as whether the visit was appropriate for a virtual encounter

- recommendations consistent with appropriate standard of care. 


\section{ATA PRACTICE GUIDELINES FOR LIVE, ON-DEMAND CARE}

Unless there is an external requirement for recording a virtual visit, the provider may opt not to record the visit. If he or she chooses to record the encounter for quality or training purposes, it shall comport with appropriate consent and privacy/security measures (see Technical Guidelines below).

B. Provider training and mentoring. Provider orientation and training should entail a thorough review of history-taking skills and physical examination skills as they pertain to the evaluation of a patient through telemedicine. Providers shall know current local and state laws as they pertain to telemedicine practice. They shall obtain the necessary training and education for themselves and/or staff to है ensure maintaining technical and clinical competence in accordance With their discipline. Providers should conduct several "dry run" " visits with test "patients" to become familiar and comfortable with the technology of virtual visits and be generally familiar with the nature of the technology the patient is using to direct and assist with $\stackrel{5}{\sim}$ minor technical questions and potential problems that may arise. The provider should also be familiar with and proficient with a satis$\underset{\sigma}{\sigma}$ factory default mode for patient engagement should technology fail ठิ during a patient encounter. Those new to telemedicine are encouraged to identify a mentor to observe during telemedicine encounters. This can also be done post hoc by a video recording. Proctored visits should include a variety of conditions and modes of encounter (e.g., phone, Web, mobile). Protocols regarding indications when care should be escalated, and provision for escalating patients when necessary to alternate modes of care should be established, documented, and communicated as part of the provider orientation process. The effectiveness of these guidelines should be assessed routinely by the provider entity as part of the standard quality review process.

\section{ETHICAL CONSIDERATIONS}

Practicing at a distance requires the same attention and adherence to professional ethical principles as would an in-person encounter. Telemedicine providers should incorporate ethical statements and $\stackrel{D}{\equiv}$ policies into their standard operating procedures.

The following are the ethical guidelines for health professionals who are engaged in telemedicine:

- A practitioner shall uphold the code of ethics for his or her profession and be aware of the codes for other professional disciplines.

- A practitioner shall abide by all federal, state, and jurisdictional laws and regulations and institutional policies.

- Telemedicine shall not be used as a means of preferentially avoiding in-person encounters based on geographic location, socioeconomic status, disease or disability, gender, gender preferences or sexual orientation, behavioral factors, ethnicity, religion, etc. An exception to this rule may be the avoidance of in-person visits during epidemics or pandemics to avoid the spread of infectious disease.

- Payment made by the patient should not be conditional on receiving a certain diagnoses or particular treatment, such as receipt of a prescription.
- Providers should abide by a strict conflict of interest policy that deters the use of telepractice for the sole purpose of enhancing income.

- Providers shall:

$\circ$ Apprise patients of their rights when receiving telemedicine, including the right to suspend or refuse treatment

$\circ$ Apprise patients of their own responsibilities when participating in telemedicine

- Inform patients of a formal complaint or grievance process to resolve ethical concerns or issues that might arise as a result of participating in telemedicine

- Discuss the potential benefits, constraints, and risks (e.g., privacy and security) of telemedicine

- Inform patients and obtain their consent when students or trainees observe the encounter.

- Providers should have a policy in place concerning the disclosure to patients of technology or equipment failures during service sessions and the contingency plans in case of technical failure and should document such events in the patient's health record.

\section{EMERGENCIES}

A. Definition of emergent conditions. An emergent condition is an illness or injury that poses an immediate threat to a person's life or long-term health. Such conditions are outside the scope of a primary and/or urgent care telemedicine practice.

B. Emergent patient evaluation and referrals. The provider shall assess a presenting patient's condition to determine severity and acuity of the patient's condition and, when indicated, refer the patient to the appropriate level of care accessible to the patient. The telemedicine provider shall be responsible for triaging the patient to the appropriate level of care (e.g., primary care provider, specialist, urgent care, emergency department). Providers may consider incorporating standard triage protocols in their telemedicine practices.

C. Documentation of emergent encounters. The provider shall document the process for treating emergent situations, which may include phoning the receiving facility in advance of the patient's arrival.

Providers shall document all referrals to emergency medical services (Dialing 911), including the medical indication/basis for the recommendation, and nature of the problem.

Providers should document the location of the patient at the start of the encounter.

Providers should document any extenuating circumstances or adverse events, be they technical or clinical, that occurred during the encounter.

Documentation should adhere to all medical-legal standards of care and, if appropriate, insurance requirements for future review and audit. 


\section{GOUGH ET AL.}

\section{FOLLOW-UP}

As noted previously, follow-up is a critical aspect of patient safety and continuity of care and should include the following:

A. Knowledge of the patient's healthcare network. The provider should have knowledge of the patient's healthcare network whenever possible, to be able to facilitate timely access to recommended specialty consultations or referrals.

B. Provision of clinical reports to referral sources. The provider, to the extent possible while being remote, shall make available relevant clinical reports to the referral institution or specialist absent a request by the patient to the contrary.

C. Transmission of home monitoring and electronic data. If feasible, the provider should facilitate transference of any home monitoring or electronic data and discuss with the patient how and if such data will be stored.

D. Patient requests for records. The provider shall establish an explicit process for patients to request copies of their telemedicine encounters at their request and to facilitate specialty care, where indicated.

\section{SPECIAL POPULATIONS}

Virtual visits can be conducted with patients with unique needs such as those with communication disorders, mental or physical disabilities, sensory disorders, or special needs related to age, gender, culture, rare diseases, or location of care. Some may need a translator or facilitator that calls for nonmedical personnel during the visit. These populations often require special considerations to ensure their engagement in the care process and follow-up and their needs are met appropriately. For instance, telemedicine providers in the United States shall be in compliance with the American Disabilities Act of 1990 and other legal and ethical requirements, described elsewhere. A useful reference to identifying and responding to these populations can be found in the ATA Practice Standards for Video-Based Online Mental Health Services. ${ }^{39}$

A. Pediatric. The literature contains examples of clinically effective pediatric telemedicine programs. ${ }^{15-20,40}$ Such pediatric encounters require the presence and/or active participation of a caregiver or facilitator, including parent/guardian, nurse, and/or childcare worker. In certain cases involving adolescents with behavioral or mental health issues, a facilitator would not remain in the room for part of or for the entire duration of the visit. Nonetheless, the practitioner shall obtain consent from the parent or legal representative of the child as required by law in the respective jurisdiction. If the parent/guardian is not present at the time of the visit, a process shall be established for prompt communication of the results of the visit with the parent/guardian.

B. Geriatric. Here again, the literature contains examples of clinically effective geriatric telemedicine programs. ${ }^{26,41}$ The evi- dence indicates frequent monitoring for chronic diseases tends to reduce the need for office visits and transportation, as well as reduce stress and increase access to care for homebound patients. ${ }^{39,42-44}$ Providers also report benefits from the ability to observe the patient in his or her home environment. ${ }^{44-49}$

In designing a system for virtual geriatric visits, providers should consider the special needs of the elderly, including vision and hearing difficulties and limited physical dexterity or mobility. These should be taken into account when designing and choosing equipment and systems. In cases where a patient demonstrates substantial confusion or anxiety during a telemedicine encounter, the practitioner should exercise judgment concerning the continuation or termination of the visit. The presence of facilitators, family members/caregivers, and nurses would facilitate the process and ultimate decision making. However, providers should have the patient affirm consent to that person's participation in the visit. A practitioner should obtain the patient's consent regarding the presence/participation of facilitators. In cases of questionable mental competency, practitioners should ensure appropriate consent from a legal proxy or representative. In circumstances where the patient is in a care facility or senior living community, a trained technician may assist in collecting relevant clinical information, including medical records, lab or diagnostic testing, and access to caregivers and staff.

In managing patients with dementia, providers should ask for the patient's durable power of attorney for healthcare decisions and use that as the legal guardian.

C. Locus of care. The literature contains examples of clinical effectiveness of successful telemedicine programs in a variety of settings, including patient homes, childcare centers, schools, chronic care facilities, the workplace, and prisons. ${ }^{6,26,27,33,36,37,41}$ All legal and regulatory requirements and ethical considerations shall be used in these settings.

\section{Technical Guidelines}

\section{SECURITY AND PRIVACY}

Providers and healthcare organizations shall comply with privacy and confidentiality requirements stipulated by HIPAA and other applicable laws. They should also familiarize themselves with security arrangements for their systems and their limitations.

This shall include appropriate disclosure to patients about sharing their personal healthcare information (PHI). Providers shall document medical records as thoroughly as if the patient participated in an in-person visit. Storage of medical records shall be accomplished using methods that are compliant with all laws pertaining to medical record storage. Access to patient information shall follow standard HIPAA privacy provisions. If an intermediary or third-party entity is engaged for the collection, storage, transmission, or processing of PHI, a Business Associate Agreement should be executed as stipulated under HIPAA.

Patients shall consent prior to any recording of the encounter, and such recording shall be available for the patients upon request. Release of such recordings data shall require written patient 


\section{ATA PRACTICE GUIDELINES FOR LIVE, ON-DEMAND CARE}

authorization or court order in a legal proceeding. Access to the recordings shall only be granted to authorized users and should be protected from accidental or unauthorized file sharing and/or transfer.

Data security shall be assured by prevailing encryption methods, including FIPS 140-2, known as the Federal Information Processing Standard. Providers should familiarize themselves with the technologies available regarding computer and mobile device security and should share such information with their patients as appropriate. Special attention should be placed on the privacy of information being communicated via mobile devices.

Mobile devices used for clinical purposes shall require authentication for access to them, as well as timeout thresholds and protections when lost or misplaced. Mobile devices should be kept in the possession of the provider when traveling or in an uncontrolled environment. Unauthorized persons shall not be allowed access to sensitive information stored on the device or use the device to access sensitive applications or network resources. Providers should have $\stackrel{5}{\infty}$ the capability to remotely disable or wipe their mobile device in the event it is lost or stolen. Videoconference software shall not allow multiple concurrent sessions to be opened by a single user. Should a second session be attempted, the system shall either log off the first session or block the second session. Session logs stored in third-party locations (i.e., not on providers' or patients' access device) shall be secure, and access to these logs shall only be granted to authorized users.

Protected health information and other confidential data shall only be backed up to or stored in secure data storage locations. Cloud services unable to achieve HIPAA compliance shall not be used for PHI or confidential data.

\section{COMMUNICATION BETWEEN ORGANIZATIONS}

Providers of telemedicine shall meet the same standards for communication between patient and provider, and between provider and other organizations, as those for in-person encounters.

\section{REMOTE MONITORING DEVICES AND DATA}

Numerous studies in the United States and elsewhere have confirmed the reliability and effectiveness of remote monitoring. ${ }^{45-49}$ This evidence reveals the benefits of remote monitoring in reducing hospitalization/re-hospitalization, greater patient compliance with medication management, timely diagnosis and initiation of treatment, and improved health outcomes.

The provider should be aware of data trends or current evidence in remote monitoring to the extent possible. Data gathered from remote monitoring should be incorporated into the visit record.

When using a personal computer (including laptops, tablets, and other mobile devices), both the provider and patient devices should, when feasible, use professional-grade or high-quality cameras and audio equipment. Devices shall have up-to-date antivirus software and, if feasible, a personal firewall installed (at least on the provider's device). Providers should ensure their personal computer or mobile device has the latest security patches and updates applied to the operating system and any third-party applications.
A. Provider organizations. Provider organizations should provide adequate resources for hardware, software, and network management, including installation, maintenance, troubleshooting, and replacement, as well as effective security arrangements. Special attention shall be paid to verify the secure and reliable networks, including successful information exchange.

B. Connectivity. Connectivity shall have adequate bandwidth, resolution, and speed for clinical consultations. Bandwidth shall be set at a minimum bandwidth of 384 kilobits/s in both the downlink and uplink directions. Resolution shall be set a minimum of $640 \times 360$, and speed at 30 frames/s. Where practical, providers may recommend preferred videoconferencing software and/or video and audio hardware to the patient. The provider and/or patient may use link test tools (e.g., bandwidth test) to pretest the connection before starting their session. Each party should use the most reliable connection method to access the Internet, including wired (e.g., Ethernet) connections when available. The videoconferencing software should be able to adapt to changing bandwidth environments without losing/dropping the connection.

In the event of a technology breakdown, causing a disruption of the session, the professional shall have a backup plan in place. The plan shall be communicated to the patient prior to commencement of the encounter, and it should be included in the general emergency management protocol.

The plan should include calling the patient via telephone and attempting to troubleshoot the issue together. It may also include referring the patient to another provider or completing the encounter by voice only.

Professionals and patients may opt to use cameras that pan, tilt, and zoom for maximal flexibility in viewing.

\section{Administrative Guidelines}

\section{VERIFICATION OF SERVICE ELIGIBILITY}

Prior to any telemedicine encounter, the provider or staff shall determine the appropriateness of telemedicine for the specific encounter and also gather information on medical history, presenting symptoms/problems, reimbursement method, and usual provider.

\section{PROVIDER AND PATIENT IDENTITY VERIFICATION}

The provider shall introduce him- or herself and any attendant personnel (e.g., residents, fellows, students) to the patient and document those present. The patient should announce those in attendance at his or her end (e.g., guardian, family). This information shall become part of the encounter document.

The full name and credentials of the provider and the full name of the patient shall be verified by birth date, address, and insurance status.

\section{PROVIDER AND PATIENT LOCATION DOCUMENTATION}

The provider shall document the location of the patient and the communication tools. The locations of the provider and patient may require documentation for reimbursement and licensing purposes. 


\section{GOUGH ET AL.}

Most states require that licensure requirements are based on the location of the patient when service is rendered. Therefore, providers shall be aware of the state where the patient is located at the time of service to assure they are licensed in that state.

Emergency management protocols are entirely dependent on where the patient receives services.

\section{CONTACT INFORMATION VERIFICATION FOR PROVIDER AND PATIENT}

Contact information shall be obtained from the patient including address of usual residence, address at time of consultation, telephone, mail, and e-mail addresses. Similarly, provider contact information shall be exchanged with patient including telephone, practice address, and e-mail. It is not necessary for the health provider to reveal his or her specific location to the patient, especially if the provider is located at home at the time of service.

\section{CREDENTIALING AND LICENSING}

All providers shall abide by the same local and regional credentialing policies as required for a traditional in-person visit as mandated by state and federal law.

Providers shall abide by all qualifications of licensure, board eligibility, or certification as required for traditional in-person visits according to by state and federal laws. The scope of care provided shall be consistent with the provider's level of training (e.g., MD/D0, ARNP, PA, RN, etc.). Providers should be cognizant of oversight requirements and auditing standards that may be applied to telemedicine patient visits as if the patient visit occurred in person. Where telemedicine/ telehealth laws require or permit different credentialing, compliance shall be maintained with those provisions.

\section{ORGANIZATIONAL POLICIES AND PROCEDURES}

Healthcare organizations should develop and implement organizational policies and procedures governing the use of telemedicine. Providers shall adhere to all applicable laws and regional and local practice as to Patient Informed Consents and Disclaimers. As part of organizational policies and procedures, healthcare entities should promulgate standards for patient and provider verification and authentication.

\section{CODING AND DOCUMENTATION}

Coding and medical record documentation should be accurate in reflecting the content of the medical visit rather than enhancing reimbursement.

Medical record and procedure coding should follow prevailing coding practices based on state and national guides such as the American Medical Association Coding Requirements.

A. Electronic medical record. Providers shall generate and maintain an electronic medical record (when feasible) for each patient for whom they provide remote care. All communications with the patient (verbal, audiovisual, or written) should be documented in the patient's unique medical record on par with documentation standards of in-person visits.
B. Access to analytics and clinical information at point of care. The provider should ensure that the patient's clinical record is available during or prior to a visit whenever possible and that sufficient time is allotted to update the patient history, if possible with the patient's primary care provider or other relevant healthcare entity.

C. Payment and billing. Prior to providing patient services, the patient shall be made aware of the patient's cost of the service to be provided, if any. Arrangement for payment should be completed prior to the delivery of the service. Special consideration must be made for Medicare patients participating in telemedicine care. Providers shall follow Medicare rules (such as the Medicare Opt Out election) for billing patients outside of standard Medicare reimbursement.

\section{Disclosure Statement}

No competing financial interests exist.

\section{REFERENCES}

1. American Telemedicine Association. Core standards for telemedicine operations May 2014. Available at www.americantelemed.org/docs/default-source/ standards/core-operational-guidelines-for-telehealth-services.pdf?sfvrsn $=6$ (last accessed January 19, 2015).

2. Alsos OA, Das A, Svanaes D. Mobile health IT: The effect of user interface and form factor on doctor-patient relationship. Int J Med Inform 2012;8:12-28.

3. Bent S, Nallamothu BK, Simel DL, Finn SD, Saint S. Does this woman have an acute uncomplicated urinary tract infection? JAMA 2002;287:2701-2710.

4. Colgan R, Williams M. Diagnosis and treatment of acute uncomplicated cystitis. Am Fam Physician 2011;84:771-776.

5. Coureya PT, Palattao KJ, Gallagher JM. HealthPartners' online clinic for simple conditions delivers savings of $\$ 88$ per episode and high patient approval. Health Aff (Millwood) 2013;32:385-392.

6. Mehrotra A. The convenience revolution for treatment of low-acuity conditions. JAMA 2013;310:35-36

7. Adamson SC, Bachman JW. Pilot study of providing online care in a primary care setting. Mayo Clinic Proc 2010;85:704-710.

8. Alverson DC, Joltz B, D'Lorio J, DeVany M, Simmons S, Poropatich RK. One size doesn't fit all: Bringing telehealth service to special populations. Telemed J $E$ Health 2008;14:957-963.

9. Barry HC, Hickner J, Ebell MH, Ettenhofer T. A randomized controlled trial of telephone management of suspected urinary tract infections in women. $\mathbf{J}$ Fam Pract 2001;50:589-594.

10. Blozik E, Sommer-Meyer C, Cerezo M, von Overbeck J. Effectiveness and safety of telemedical management in uncomplicated urinary tract infections. J Telemed Telecare 2011;17:78-82.

11. Demaris G, Speedie SM, Finkelstein S. Change of patients' perceptions of TeleHomeCare. Telemed J E Health 2001;7:241-248.

12. Dixon RF, Stahl JE. Virtual visits in a general medicine practice: A pilot study. Telemed J E Health 2008;14:525-530.

13. Department of Justice, Office of Justice Programs, National Institute of Justice. Telemedicine can reduce correctional health care costs: An evaluation of a prison telemedicine network. Research Report. March 1999. Available at https://www.ncjrs.gov/pdffiles1/175040.pdf (last accessed January 19, 2015).

14. Vitacca M, Bianchi L, Guerra A, Fracchia C, Spanevello A, Balbi B, Scalvini S. Tele-assistance in chronic respiratory failure patients: $A$ randomized clinical trial. Eur Respir J 2009;33:411-418.

15. American Telemedicine Association. Practice guidelines for video-based online mental health services, pp. 15-18. May 2013. Available at www.american 


\section{ATA PRACTICE GUIDELINES FOR LIVE, ON-DEMAND CARE}

telemed.org/resources/telemedicine-practice-guidelines/telemedicine-practiceguidelines/practice-guidelines-for-video-based-online-mental-health-services \#.VLAPVnsONEw (last accessed January 19, 2015).

16. Herendeen NE, Deshpande P. Telemedicine and the patient-centered medical home. Pediatr Ann 2014;43:e28-e32.

17. Herendeen NE, Schaefer GB. Practical applications of telemedicine for pediatricians. Pediatr Ann 2009;38:567-569.

18. McConnochie KM, Conners GP, Brayer AF, Goepp J, Herendeen NE, Wood NE, Thomas A, Ahn DS, Roghmann KJ. Effectiveness of telemedicine in replacing in-person evaluation for acute childhood illness in office settings. Telemed J E Health 2006;12:308-316.

19. McConnochie KM, Conners GP, Brayer AF, Goepp J, Herendeen NE, Wood NE, Thomas A, Ahn DS, Roghmann KJ. Differences in diagnosis and treatment using telemedicine versus in-person evaluation of acute illness. Ambul Pediatr 2006;6:187-195; discussion 196-197.

20. McConnochie KM, Wood NE, Kitzman HJ, Herendeen NE, Roy J, Roghmann KJ. Telemedicine reduces absence resulting from illness in urban child care: Evaluation of an innovation. Pediatrics 2005;1215:1273-1282.

21. McConnochie KM, Tan J, Wood NE, Herendeen NE, Dick A, Kitzman H, Roghmann K. Acute illness utilization patterns before and after telemedicine in childcare for inner-city children: A cohort study. Telemed J E Health 2007;13:381-390.

22. Federation of State Medical Boards. Model policy for the appropriate use of telemedicine technologies in the practice of medicine. April 2014. Available at www.fsmb.org/Media/Default/PDF/FSMB/Advocacy/FSMB_Telemedicine_ Policy.pdf (last accessed January 19, 2015).

23. American Medical Association. Report of the Council on Medical Service: Coverage of and payment for telemedicine. CMS Report 7-A-14. June 2014. Available at www.jonesday.com/files/upload/AMA\%20Policy\%20on\%20 Telehealth\%20\%28June\%202014\%29.PDF (last accessed January 19, 2015).

24. Schmidt S, Schuchert A, Krieg T, Oeff M. Home telemonitoring in patients with chronic heart failure: A chance to improve patient care? Dtsch Arztebl Int 2010;107:131-138.

25. Bennett, SJ, Hays LM, Embree JL, Arnould M. Heart Messages: A tailored message intervention for improving heart failure outcomes. $J$ Cardiovasc Nurs 2000;14:94-105

26. Gellis ZD, Kenaley B, McGinty J, Bardelli E, Davitt J, Ten Have T. Outcomes of a telehealth intervention for homebound older adults with heart or chronic respiratory failure: A randomized controlled trial. Gerontologist 2012;52:541-552.

27. Goulis DG, Giaglis GD, Boren SA, Lekka I, Bontis EA, Maglaveras N, Avramides A. Effectiveness of home-centered care through telemedicine applications for overweight and obese patients: A randomized controlled trial. Int J Obes 2004; 28:1391-1398.

28. Graham AL, Cobb NK, Papandonatos GD, Moreno JL, Kang H, Tinkelman DG, Bock BC, Niaura RS, Abrams DB. A randomized trial of internet and telephone treatment of smoking cessation. Arch Intern Med 2011;171:46-53.

29. Kashim A, Droogan MT, Santamore WP, Wald JW, Marble JF, Cross RC, Bove AA. Web-based internet telemedicine management of patients with heart failure. Telemed J E Health 2006;12:439-447.

30. Mehrotra A, Paone S, Martich GD, Albert SM, Shevchik GJ. Comparison of care at e-visits and physician office visits for sinusitis and urinary tract infection. JAMA Intern Med 2013;173:72-74.

31. Marziali E. E-health program for patients with chronic disease. Telemed J E Health 2009;15:176-181.

32. Moth G, Huibers L, Christensen MB, Vedsted P. Drug prescription by telephone consultation in Danish out-of-hours primary care: A population-based study of frequency and associations with clinical severity and diagnosis. BMC Fam Pract 2014;15:142.

33. Hughes CL, Marshall CR, Murphy E, Mun SK. Technologies in the patientcentered medical home: Examining the model from an enterprise perspective. Telemed J E Health 2011;17:495-500
34. Kraschnewski JL, Gabbay RA. Role of health information technologies in the patient-centered medical home. J Diabetes Sci Technol 2013;7:13761385.

35. Agency for Healthcare Research and Quality. Defining the PCHM. Available at www.pcmh.ahrq.gov/page/defining-pcmh (last accessed January 19, 2015).

36. Larsen D, Stamm BH, Davis K, Magaletta PR. Prison telemedicine and telehealth utilization in the United States: State and federal perceptions of benefits and barriers. Telemed J E-Health 2004;10(Suppl 2):S81-S89.

37. Ohinmaa A, Chatterley $P$, Nguyen $T$, Jacobs $P$. Telehealth in substance abuse and addiction: Review of the literature on smoking, alcohol, drug abuse and gambling. 2010. Available at www.ihe.ca/documents/Telehealth\%20in\%20 Addiction-Final\%202\%20Arto.pdf (last accessed January 19, 2015).

38. Raimer BG, Stobo JD. Health care delivery in the Texas prison system: The role of academic medicine. JAMA 2004;292:485-489.

39. American Telemedicine Association. Practice standards for video-based online mental health services. ATA Standards and Guidelines. May 2013. Available at www.americantelemed.org/resources/telemedicine-practice-guidelines/ telemedicine-practice-guidelines/practice-guidelines-for-video-based-onlinemental-health-services\#.VLAfkHsONEw (last accessed January 19, 2015).

40. Spooner SA, Gotlieb EM. Telemedicine: Pediatric application. Pediatrics 2004;113:e639-e643.

41. Shah MN, McDermott R, Gillespie SM, Philbrick EB, Nelson D. Potential of telemedicine to provide acute medical care for adults in senior living communities. Acad Emerg Med 2013;10:162-168.

42. Amaert A, Delesie L. Telenursing for the elderly. The case for video-telephony. J Telemed Telecare 2001;7:311-316.

43. Shauberger C, Merkitch KW, Prell AM. Acute cystitis in women: Experience with a telephone-based algorithm. WMJ 2007;106:326-329.

44. Unwin BK, Jerant AF. The home visit. Am Fam Physician 1999;60:1481-1488.

45. Anker SD, Koehler F, Abraham WT. Telemedicine and remote management of patients with heart failure. Lancet 2011;378:731-739.

46. Antonicelli R, Testarmata P, Spazzafumo L, Gagliardi C, Bilo G, Valentini M, Olivieri $F$, Parati $G$. Impact of telemonitoring at home on the management of elderly patients with congestive heart failure. J Telemed Telecare 2008;14:300305.

47. Dang S, Dimmick S, Kelkar G. Evaluating the evidence base for the use of home telehealth remote monitoring in elderly with heart failure. Telemed J E Health $2009 ; 15: 783-796$

48. Paré G, Mogadem K, Pineau G, St-Hilaire C. Clinical effects of home telemonitoring in the context of diabetes, asthma, heart failure and hypertension: A systematic review. J Med Internet Res 2010;16:e21.

49. Seto E, Leonard KJ, Cafazzo JA, Barnsley J, Masino C, Ross HJ. Perceptions and experiences for heart failure patients and clinicians on the user of mobile phone-based telemonitoring. J Med Internet Res 2012;10;e25.

Address correspondence to: Sunil Budhrani, $M D, M P H, M B A$ CareClix

1934 Old Gallows Road, Suite 350

Tysons Corner, VA 22182

E-mail: sunilermd@yahoo.com

Received: January 13, 2015

Revised: January 13, 2015

Accepted: January 13, 2015 


\section{This article has been cited by:}

1. Tan Lo Fu, Mason Natalie, Gonzaga W. James. 2017. Virtual Visits for Upper Respiratory Tract Infections in Adults Associated with Positive Outcome in a Cox Model. Telemedicine and e-Health 23:3, 200-204. [Abstract] [Full Text HTML] [Full Text PDF] [Full Text PDF with Links]

2. Johan Verbraecken. 2016. Telemedicine Applications in Sleep Disordered Breathing. Sleep Medicine Clinics 11:4, 445-459. [Crossref]

3. Joao M. Alves, Danielle B. L. Albino, Marisete C. Resener, Marlene Zannin, Alexandre Savaris, Christiane G. von Wangenheim, Aldo von Wangenheim. Quality Evaluation of Poison Control Information Systems: A Case Study of the DATATOX System 30-35. [Crossref]

4. Bollepalli S. Chandra, Challa S. Sastry, Soumya Jana. 2015. Reliable resource-constrained telecardiology via compressive detection of anomalous ECG signals. Computers in Biology and Medicine 66, 144-153. [Crossref] 